\title{
A Review of mTOR Pathway Inhibitors in Gynecologic Cancer
}

\author{
Andréia Cristina de Melo, Eduardo Paulino, and Álvaro Henrique Ingles Garces
}

Brazilian National Cancer Institute (INCA), Rio de Janeiro, RJ, Brazil

Correspondence should be addressed to Andréia Cristina de Melo; melo.andreia@uol.com.br

Received 3 November 2016; Accepted 12 January 2017; Published 13 February 2017

Academic Editor: Yasuhiro Maejima

Copyright (c) 2017 Andréia Cristina de Melo et al. This is an open access article distributed under the Creative Commons Attribution License, which permits unrestricted use, distribution, and reproduction in any medium, provided the original work is properly cited.

\begin{abstract}
The treatment of advanced gynecologic cancers remains palliative in most of cases. Although systemic treatment has entered into the era of targeted drugs the antitumor efficacies of current therapies are still limited. In this context there is a great need for more active treatment and rationally designed targeted therapies. The PI3K/AKT/mTOR is a signaling pathway in mammal cells that coordinates important cell activities. It has a critical function in the survival, growth, and proliferation of malignant cells and was object of important research in the last two decades. The mTOR pathway emerges as an attractive therapeutic target in cancer because it serves as a convergence point for many growth stimuli and, through its downstream substrates, controls cellular processes that contribute to the initiation and maintenance of cancer. Aberrant PI3K-dependent signaling occurs frequently in a wide range of tumor types, including endometrial, cervical, and ovarian cancers. The present study reviewed the available evidence regarding the potential impact of some mTOR pathway inhibitors in the treatment of gynecological cancer. Few advances in medical management have occurred in recent years in the treatment of advanced or recurrent gynecological malignancies, and a poor prognosis remains. Rationally designed molecularly targeted therapy is an emerging and important option in this setting; then more investigation in $\mathrm{PI} 3 \mathrm{~K} / \mathrm{AKT} / \mathrm{mTOR}$ pathway-targeted therapies is warranted.
\end{abstract}

\section{Introduction}

The treatment of advanced gynecologic cancers remains palliative in most of cases and the vast majority of the patients will eventually die. Although systemic treatment has entered into the era of targeted drugs the antitumor efficacies of current therapies are still limited, most likely because of the high degree of cancer clonal heterogeneity and cell signal complexity [1]. In this context there is a great need for more active treatment and rationally designed targeted therapies [2].

The $\mathrm{PI} 3 \mathrm{~K} / \mathrm{AKT} / \mathrm{mTOR}$ is a signaling pathway in mammal cells that coordinates important cell activities [2]. It has a critical function in the survival, growth, and proliferation of malignant cells and was object of important research in the last two decades [3-5]. The deregulation of the mammalian target of rapamycin (mTOR) and other proteins of this pathway occurs in many solid tumors and tumor cells have more sensitivity to mTOR inhibitors than normal cells [6]. Mechanisms for pathway activation include loss of tumor suppressor PTEN (phosphatase and tensin homolog) function, amplification or mutation of PI3K (phosphoinositide 3-kinase), amplification or mutation of $A K T$ (protein kinase $B$ ), activation of growth factor receptors, and exposure to carcinogens $[7,8]$.

The mTOR pathway emerges as an attractive therapeutic target in cancer because it serves as a convergence point for many growth stimuli and, through its downstream substrates, controls cellular processes that contribute to the initiation and maintenance of cancer [8]. Aberrant PI3K-dependent signaling occurs frequently in a wide range of tumor types, including endometrial, cervical, and ovarian cancers $[2,9]$.

\section{Endometrial Cancer}

Endometrial cancer (EC) is the most common and the second cause of death among gynecologic cancers in United States, with more than 60.000 new cases and 10.000 deaths expected in 2016 [10]. Unfortunately, data from 2013 [11] shows that EC research received far less funding than ovarian cancer (\$17.8 versus \$100.8 million, resp.) and this uneven funding 
translates in almost four times less research projects for EC compared to ovarian cancer (488 versus 1785, resp.) [12].

Initial approach to EC is surgical staging with hysterectomy plus salpingoforectomy, with or without lymph node assessment. Adjuvant treatment is based on risk factors (FIGO stage, histology, grade, etc.) and nowadays patients are receiving more systemic treatment upfront, even in early stage disease $[13,14]$. For those with advanced and recurrent disease, treatment options are much more limited, with a doublet of platinum salt and taxane for first-line treatment and no standard approach for future lines of therapy.

Historically, EC was divided into type I (mainly endometrioid histology) and type II (nonendometrioid) carcinomas but this classification does not take into account the molecular profiles of tumors [15]. In the last decade more attention has been given to molecular pathways and like many other types of cancers target therapy emerged as an excellent option of treatment. In TCGA project for EC [16] (mainly endometrioid and serous histology) four molecular subgroups of EC were seen: POLE-ultramutated, MSI-hypermutated, copy number high (serous-like), and copy number low, with each subgroup showing different altered molecular pathways.

$\mathrm{PI} 3 \mathrm{~K} / \mathrm{AKT} / \mathrm{mTOR}$ is the most important altered pathway in EC and it seems to harbor the highest alterations among all solid tumors. Oza et al. [17] reported that this pathway could be target with mTOR inhibitor (temsirolimus) and it became one of the milestones in EC. Since that many trials were published targeting $\mathrm{PI} 3 \mathrm{~K} / \mathrm{AKT} / \mathrm{mTOR}$ pathway with promising results.

2.1. PI3K/AKT/mTOR Pathway and Endometrial Cancer. The TCGA reported in 2013 the molecular profile of EC [16]. It collected data from 373 patients with endometrioid and serous adenocarcinoma (clear cell was not represented). A genomic, transcriptomic, and proteomic analysis was done using array and sequencing-based technologies and four major molecular profiles were found: POLE (ultramutated), MSI (hypermutated), copy number low (CNL, endometrioid), and copy number high ( $\mathrm{CNH}$, serous-like), with distinct outcomes in progression-free survival (PFS) (POLE and $\mathrm{CNH}$ subgroups have the highest and poorest outcome, resp., $[p=0.02])$. In general, EC has alterations on PI3KCA, PIK3R1, AKT1, and PTEN in about 59.7\%, 33\%, $3.2 \%$, and $66 \%$ of cases, respectively. When subgroups are analyzed separated, POLE has the highest rate of PTEN, PI3KCA, and PIK3R1 alterations: 94\%, 71\%, and 65\%, respectively. On the other side, $\mathrm{CNH}$ has different type of mutations/amplifications, with $P 53$ being the most altered in $92 \%$ of the EC (PI3K 47\% and PTEN 11\%). Also, CNH showed HER2 amplifications in $25 \%$ of EC.

2.2. Therapeutics with $P I 3 K / A K T / m T O R$ Inhibitors. There are many drugs being tested in each part of the pathway: inhibiting PI3K, mTOR, AKT, and dual inhibitors on $\mathrm{PI} 3 \mathrm{~K} / \mathrm{mTOR}$ and PI3K/AKT. Until now the most tested drugs are those blocking mTOR activity. Oza et al. [17] showed promising results with temsirolimus in 54 recurrent or metastatic EC patients with no $(n=29)$ or prior $(n=$
25) lines of therapy. Chemotherapy-naïve patients had $14 \%$ of response rate (RR), comparing to $4 \%$ on chemotherapytreated group. A recent randomized phase 2 study compared single-agent ridaforolimus to progestins or investigator's choice chemotherapy in 130 patients with advanced EC and prior systemic treatments [18]. Patients in ridaforolimus arm had higher PFS $(3.6 \times 1.9$ months, $p=0.008)$ and more stable disease (SD) (35\% versus $27 \%, p=0.021)$, but also more grade $3 / 4$ adverse events like diarrhea (11.1\% versus $1.5 \%)$, hyperglycemia (19\% versus $0 \%)$, and anemia (12.7\% versus 4.6\%). Many other trials have been testing mTOR inhibitors to date and taking together these trials showed moderate activity with overall response rate (ORR) varying from 4 to $24 \%$ (with higher responses for those chemotherapy-naïve) and prolonged SD [19-23]. Toxicity profile was manageable with the most reported events being hematological, metabolically, constitutional, and gastrointestinal. PI3K and AKT inhibitors are also being studied. Pilaralisib [24], an PI3K inhibitor, showed minimal efficacy in recurrent EC patients who received prior systemic therapy, with RR of $6 \%$ and SD of $37.3 \%$, and the AKT inhibitor MK-2206 [25] showed minimal activity with $5.5 \% \mathrm{RR}$ and $33 \% \mathrm{SD}$ in 36 patients with prior systemic therapies for recurrent EC with high rate of grade $3 / 4$ adverse events (58\%).

Emerging strategies are trying to combine drugs that have different ways of action in order to overcome resistance and some studies have shown the feasibility of this strategy. On a phase 2 trial [26], patients were randomized between temsirolimus with or without megestrol acetate alternating with tamoxifen. This study reported no improvement on efficacy and unfortunately closed early due to excess of venous thrombosis. Promising clinical activity was seen combining everolimus plus anastrozole. Slomovitz et al. [27] showed an ORR of $32 \%$ in patients with prior systemic therapies who were treated with this combination, and the responses were even higher in those women who were taking metformin for diabetes (ORR of 55\%). Based on these astonishing results with the triplet regimen, the authors planned another phase II trial and presented the results at ASCO 2016 [28]. Patients treated with the combination of everolimus, anastrozole, and metformin had an ORR of $29 \%$, with $60 \%$ having clinical benefit rate [complete response $(\mathrm{CR})+$ partial response $(\mathrm{PR})$ $+\mathrm{SD}$ ]. Antiangiogenic agents also showed to be active on EC (mainly bevacizumab) and studies combining these drugs with mTOR inhibitors were designed and tested. Alvarez et al. [29] reported that in pretreated EC this combination demonstrated an ORR of $25 \%$, but with high rate of toxicities (39\% of patients discontinued treatment for toxicity). Einstein et al. [30] reported the results of this combination on pretreated EC and unfortunately this study did not meet the prespecified efficacy criteria. They reported PR and SD in $20 \%$ and $48 \%$ of patients at 6 months, respectively.

Recently, Aghajanian et al. [31] presented a randomized phase II trial, comparing carboplatin and paclitaxel with either temsirolimus or bevacizumab or carboplatin plus ixabepilone and bevacizumab to the historical control arm of GOG 209 (carboplatin and paclitaxel arm). This study showed improved overall survival (OS) for the antiangiogenic therapy when added to carboplatin and paclitaxel backbone, 
but unfortunately temsirolimus did not improve outcomes compared to historical control arm.

Based on some studies suggesting that blocking the $\mathrm{PI} 3 \mathrm{~K} / \mathrm{AKT} / \mathrm{mTOR}$ pathway could interfere with and cause defects in the DNA repair mechanism [32], like homologous recombination, much interest has emerged combining these pathway inhibitors with PARP inhibitors [33]. On cells with DNA double-strand breaks, PTEN loss may dysfunct the homologous recombination repair, "mimicking" BRCA1/2 mutation. Combining PI $3 \mathrm{~K} / \mathrm{AKT} / \mathrm{mTOR}$ and PARP inhibitors could recapitulate the synthetic lethality observed in ovarian cancer patients with BRCA germ line mutation treated with olaparib. Gathering the high rate of PI3K alterations and PTEN loss in EC, it seems this is a promising strategy in this neoplasia. One recent case [34] showed the activity of olaparib in a patient with metastatic endometrial cancer, BRCA negative and heavily pretreated with multiple lines of platinum compound, taxane and doxorubicin. This patient showed PR in liver, lung, and brain lesions.

Recent progress on immune mediators in oncology brought a lot of hope of better and more efficient drugs to treat cancer. And this fact is not different for gynecologic cancers. Some evidences emerged showing the importance of mTOR in optimizing the immune response. It has a role in antigen presentation by dendritic cells as well as promoting expression of CD86 (stimulatory molecules) and decreasing PDL1 on T cells (inhibitory molecules). Based on this fact, some phase I studies are evaluating the role of mTOR inhibitors with therapeutic vaccines (NCT01522820).

\section{Cervical Cancer}

Cervical cancer (CC) is a public health problem, representing the fourth most commonly diagnosed cancer and the seventh overall, with an estimated 528,000 new cases in 2012 across the world [35]. There were an estimated 266,000 deaths from CC worldwide in 2012, accounting for $7.5 \%$ of all female cancer deaths and this number is expected to increase up to 410,000 by 2030 [36]. Each year, approximately 200,000 women die of this disease. Developing countries account for approximately 76 to $85 \%$ of CC cases [36].

Virtually all CCs (more than 99\%) are caused by high-risk human papillomavirus (HPV) [37]. The HPV E7 oncoprotein is essential for CC carcinogenesis. The AKT phosphorylation demonstrated in samples of CC suggests a constitutive activation of the PI3K/AKT pathway in patients [38]. Moreover, mTOR inhibitors block the 4E-BP1-protein phosphorylation and significantly reduce the level of E7 protein on in vitro models, leading to an accumulation of cells on G1 phase and thereby inducing apoptosis [39]. In addition, it is established that radiation activates the PI3K/AKT pathway and mTOR inhibitors sensitize tumor and endothelial cells to cisplatin and radiotherapy effects [40].

Evaluating locally advanced CC, de Melo et al. [41] recently published the results of a phase I trial combining everolimus to the standard treatment. In a $3+3$ design the trial aimed to treat 3 dose levels of at least 3 patients with orally daily doses of everolimus ( $2.5,5$, and $10 \mathrm{mg} /$ day), cisplatin, and radiotherapy delivered in a 9-week interval in CC patients, stages IIB, IIIA, or IIIB. Patients received everolimus from day 7 up to the last day of brachytherapy. Primary objective was to evaluate safety, toxicity, and the maximum tolerated dose (MTD) of everolimus in association with cisplatin and radiotherapy. Pharmacokinetic (PK) parameters and response rates were analyzed as secondary objectives. Thirteen patients were enrolled, 6 at $2.5 \mathrm{mg}, 3$ at $5 \mathrm{mg}$, and 4 at $10 \mathrm{mg}$ of everolimus. Four patients did not complete the planned schedule, 1 at $2.5 \mathrm{mg}$ presented grade 4 acute renal failure interpreted as dose limiting toxicity (DLT) and 3 at $10 \mathrm{mg}: 1$ with disease progression and 2 with DLTs, 1 grade 3 rash and 1 grade 4 neutropenia. PK results were characterized by dose-dependent increases in AUC and Cmax. Response assessment was done 12 weeks after the end of treatment and 12 patients were evaluable for response. Eleven out of 12 evaluable patients (91.6\%) experienced CR and $1(8.4 \%)$ experienced PR, with ORR of $100 \%$ at the end of treatment according to RECIST 1.1. Using the metabolic response assessment (PET/CT), 9 (75\%) patients had CR and 3 (25\%) had PR.

In a different scenario of patients with metastatic, persistent, or recurrent disease, a nonrandomized phase 1 clinical trial [42] evaluated 74 patients with gynecologic and breast malignancies treated with liposomal doxorubicin, bevacizumab, and temsirolimus. Thirteen CC patients were included and 10 had squamous cell carcinoma. Primary endpoints were to establish the MTD and characterize DLTs with a preliminary assessment of antitumor efficacy as secondary endpoint. All 74 patients included were heavily pretreated with a median of 4 previous chemotherapy lines. Two PR in the group of CC patients with squamous cell carcinoma (treated with dose level 6: bevacizumab $15 \mathrm{mg} / \mathrm{kg}$ IV day 1 , liposomal doxorubicin $30 \mathrm{mg} / \mathrm{m}^{2}$ IV day 1 , and temsirolimus $25 \mathrm{mg}$ IV days 1,8 , and 15) were detected. The MTD for the study was reached at level 6 . The recommended dose (RD) for phase 2 trial was bevacizumab of $15 \mathrm{mg} / \mathrm{kg}$ at day 1 , liposomal doxorubicin of $20-30 \mathrm{mg} / \mathrm{m}^{2}$ at day 1 , and temsirolimus of $25 \mathrm{mg}$ IV at days 1,8 , and 15 . The ORR in this heavily pretreated population was $20.3 \%$. All $74(100 \%)$ patients experienced at least 1 adverse event, mostly reversible grade 1 or grade 2, possibly drug related. Treatment combination was relatively safe and well tolerated. Among the 15 responders (CR + PR), PIK3CA and PTEN status were known in $9(60 \%)$ and $5(33.3 \%)$, respectively. Four $(44.4 \%)$ of the 9 responders for whom PI3KCA mutational status was known were positive and $3(60 \%)$ of the 5 responders for whom PTEN status was known were found to have PTEN loss.

Piha-Paul et al. [43] evaluated in a phase 1 trial 41 patients with advanced gynecologic malignancies treated with bevacizumab and temsirolimus. Six patients with CC were included and 4 had squamous cell carcinoma. Primary endpoints were to establish the MTD and characterize DLTs; a preliminary assessment of antitumor efficacy was the secondary endpoint. All 41 patients were heavily pretreated with a median number of 4 previous lines of chemotherapy. Among all patients included, $20 \%$ had SD lasting more than 6 months. Analysis of mutational status of PTEN, PI3K, RAS, 
and $R A F$ was not performed in all included patients. In 2 patients who have PRs, the mutational status was not done in one and the other patient has negative status mutations for $P I 3 K, R A S$, and RAF. The five responders for whom PTEN status was known were found to have PTEN loss. The highest dose escalation was obtained (dose level 13: bevacizumab of $15 \mathrm{mg} / \mathrm{kg} \mathrm{IV}$ at day 1 and temsirolimus of $25 \mathrm{mg} / \mathrm{kg}$ IV at days 1,8 , and 15) and MTD was not reached. All 41 patients experienced at least one adverse event that was possibly drug related. These events were mostly grade 1 or grade 2 and reversible; $71 \%$ of the patients experienced no treatmentrelated toxicity greater than grade 2 .

In a nonrandomized phase 2 clinical trial Tinker et al. [44] evaluated 38 patients with CC. Primary endpoint was the objective RR as determined by RECIST version 1.1. Up to one prior line chemotherapy for metastatic or recurrent disease was allowed. Patients were treated with temsirolimus of $25 \mathrm{mg}$ IV weekly in 4-week cycles. Only one patient with cervical adenocarcinoma had a PR. The median duration of SD was 6.5 months (range 2.4-12.0), 28\% lasting 6 months or more (95\% CI: 14-43\%). The median PFS was 3.52 months (95\% CI: 1.81-4.7). Eleven serious adverse events among 7 patients that were possibly related to the protocol therapy were observed. No significant association was found with any of the markers tested and response or progression on temsirolimus therapy

Due to the results of these 3 studies in a heavily pretreated population the effectiveness of therapy is not clear, and there is a tendency of activity using mTOR inhibitor treatment, but more clinical trials are needed.

\section{Ovarian Cancer}

Ovarian cancer (OC) is the most important cause of death among gynecologic cancers and, in women, it is the sixth most common cancer worldwide [45-47]. Only in the United States, 22,280 new cases of OC and 14,240 deaths were estimated for 2016 [48]. In Europe, 65,538 new cases of OC were expected for 2014 and 42,716 women were estimated to die due to this disease $[49,50]$. Unfortunately, more than $60 \%$ of women with OC are diagnosed in advanced stages. The first-line treatment for patients diagnosed with OC is optimal cytoreductive surgery followed by a combination of chemotherapy with platinum and paclitaxel: this is the basis and the first choice for OC treatment [51]. The response to platinum-based chemotherapy is excellent upfront, even in high-grade serous ovarian cancer (HGSOC) [52-54], but approximately $25 \%$ of these patients acquire de novo resistance during primary treatment or relapsing within 6 months (more than $50 \%$ of the responders will have recurrence of disease) $[52,53,55]$.

Patients who have PD during platinum-based chemotherapy (platinum-refractory) or with a progression within 6 months after completion of platinum-based chemotherapy (platinum-resistant) have a poor prognosis even with standard second-line therapies [49, 56-58]. Although the inclusion of bevacizumab to the chemotherapy could improve PFS for patients with platinum-resistant OC [59] there is still an urgent need to develop novel treatments based on the distinct biological background of this disease $[49,53]$.
Nowadays, due to a wide range of molecular profiling studies, that is, the genomic analyses conducted by the Cancer Genome Atlas (TCGA) network, the knowledge and comprehension of the molecular pathogenesis of OC have improved $[51,53,60]$. It is unquestionable that $\mathrm{OC}$ is an extremely heterogeneous disease with important differences not only in molecular profile and histology but also in prognosis and chemosensitivity, depending on the subtype [51, 61]. HGSOC, the most common subtype of OC, has as important characteristic, genomic instability, as well as sensitivity to platinum-based chemotherapy. Apart from P53 mutations, which are present in $98 \%$ of cases, gene mutations that are frequently identified in other solid tumors (i.e., PIK3CA) are not usually found in HGSOC $[51,62]$.

Clear cell, endometrioid, low-grade serous, transitional, and mucinous subtypes are examples of uncommon subtypes of epithelial OC. Different from HGSOC, these rare subtypes show, very frequently, oncogenic mutations (which could be target of novel therapies), besides genomic stability. They have better prognosis when compared to HGSOC due to the fact that their diagnosis is often done in initial stages, but at the same time, chemoresistance is a recognized characteristic of the advanced and recurrent disease [51, 63].

Oftentimes, the PI3K/AKT/mTOR pathway is deregulated in $\mathrm{OC}$ and PIK3CA mutations have been reported in approximately $12 \%$ of OCs [64-66]. The mTOR pathway is activated in approximately $70 \%$ (activated in about $50 \%$ of the HGSOC patients) [66, 67]. It is interesting that the type of PI3K alteration seems to be related to the histology. PTEN loss has been identified in 5\% of cases of HGSOC and amplifications in PIK3CA in 20\% and in one of the AKT isoforms (AKT1, AKT2, and AKT3) in 10\%-15\% of cases $[51,67]$.

The levels of expression of phosphorylated AKT (pAKT) and PIK3CA were found to be related to a lower survival rate and the activation of the pathway was found to be an independent negative prognostic marker in OC (measured by AKT or mTOR phosphorylation levels) [51, 68]. Preclinical experimental studies also suggested that concomitant mutations in the MAPK pathway (KRAS, NRAS, and BRAF) can mediate resistance to therapy and PIK3CA mutations can predict response to PI3K and/or mTOR inhibitors $[65,69,70]$.

A recent paper presented in ASCO Meeting 2016 evaluated 379 patients with ovarian or fallopian tube cancer. The frequency of alterations in 10 genes (AKT1, AKT2, AKT3, mTOR, PIK3CA, PIK3C2B, PIK3R1, PTEN, TSC1, and TSC2) in the PTEN/PI3K/AKT/mTOR pathway was evaluated and determined. It was demonstrated that $33 \%$ of the OC patients have aberrations in at least one of the ten selected genes and the most common alterations were PIK3CA in $12 \%$ of the patients, followed by PTEN alterations in $10 \%$ and AKT2 amplifications in 2\%. Missense mutations in $m T O R$, TSC1, TSC2, and PIK3C2B were also identified and TP53 missense/nonsense mutations were found in $70 \%$ of the cases [70].

Some tumor types have shown response when treated with mTOR inhibitors (temsirolimus, everolimus, and ridaforolimus) and this class of drugs has also been assessed in OC. 
4.1. mTOR Inhibitors: Monotherapy. GOG 1701 is a phase II clinical trial that evaluated the use of temsirolimus in OC patients. Fifty-four patients were enrolled and five of them (5.3\%) presented PR to monotherapy for refractory, recurrent OC, or primary peritoneal cancers [71].

In another phase II trial published in 2016, OC patients were treated with weekly temsirolimus at a flat dose of $25 \mathrm{mg}$. Objective responses were observed in $9.3 \%$ of patients and the 6 -month PFS rate was $24 \%$; hence, the study failed to meet its efficacy endpoint. However, a few patients in this study had long lasting remissions or disease stabilization under mTOR-inhibition treatment [49]. The authors concluded that the observed activity was insufficient to justify a phase III trial of temsirolimus in unselected OC patients. The trial included mainly serous tumors and only a few endometrioid (4 of 54,7\%) or clear cell ovarian tumors ( 3 of 54, 6\%), the two subtypes most likely to demonstrate PIK3CA mutations. Interestingly, 1 of the 3 clear cell OCs had an objective PR to temsirolimus. More recently, one objective response lasting for 14 months and $1 \mathrm{SD}$ was reported for 5 patients with clear cell OC treated with temsirolimus [72].

4.2. mTOR Inhibitors: Combination Therapy. Considering the limited activity of mTOR inhibitors as monotherapy and the evidence from preclinical studies indicating an additional benefit of mTOR inhibitors when associated with chemotherapy, some trials have investigated the effects of the combination of mTOR and cytotoxic drugs [51]. Phase I studies evaluating patients with gynecological tumors and treated with temsirolimus combined with weekly topotecan or pegylated liposomal doxorubicin (PLD) have been performed. However, those studies showed no expressive results as they were small and the activity was limited [19, 51, 73]. Another phase I clinical trial evaluating the use of temsirolimus plus carboplatin/paclitaxel $[51,74]$ showed PR in 3 of 6 patients with OC. Other two phase I studies have been reported, one combining ridaforolimus plus carboplatin and paclitaxel $[51,75]$ and another one associating everolimus with weekly paclitaxel $[51,76]$.

A phase II study evaluated a total of 140 patients with advanced breast, CC, EC, and OC when treated with mTOR inhibitors. Sixty patients (43\%) had OC and PIK3CA mutations were detected in $12 \%$ of them. There was no significant association of PIK3CA mutation status with age, disease type, or ethnicity. Responses were observed only in the combination schedules, but not with monotherapy (44\% versus $0 \%$, resp., $p=0.06$ ) [65].

Another phase II trial, now assessing the efficacy of temsirolimus and trabectedin in patients with recurrent clear cell carcinoma, was performed. In this study, 17 patients were enrolled and the ORR was $18 \%$ [CR $=1$ patient; SD $>3$ months $=5$ patients (29\%)] [51, 77]. Considering all the information above, it is difficult to establish final conclusions regarding the importance of the association of mTOR inhibitors and cytotoxic drugs [51]. Currently, there are ongoing trials recruiting patients evaluating the use of MTOR inhibitors (i.e., mTORC1/2 inhibitor AZD2014 or the oral AKT inhibitor AZD5363 for recurrent endometrial, NCT02208375; ovarian cancer and a phase I trial of the combination of AZD2014 and weekly paclitaxel, NCT02193633) and in view of this new data, this question might be clarified.

As previously stated, phases I and II trials in recurrence or refractory OC have shown a modest response, but the presence of confounding factors suggests that further investigation is needed [45]. Intensive research on the $\mathrm{PI} 3 \mathrm{~K} / \mathrm{AKT} / \mathrm{mTOR}$ pathway and its interactions with other cellular signaling mechanisms is necessary and simple solutions are not likely to be expected $[49,78]$.

The combination of endocrine therapy with PI3K inhibition in low-grade serous and endometrioid ovarian tumors would also be of considerable relevance as these subtypes frequently express hormone receptors $[23,45]$. The association of MEK and PI3K inhibitors could also be appropriate in lowgrade serous OC based on the rational that this subgroup presents frequent alterations in the RAS and PI3K pathways [51]. When proposing new therapies, it is also important to consider target specificity of the drug, that is, sirolimus, whose target is limited to the mTORC1 $[51,67]$.

In summary, advances in genomic characterization of OC have been seen without corresponding successful targeted therapies [51,65]. The role of mTOR inhibitors is not completely clear or defined, especially in the context of combination therapy, which may be antagonistic or a chemoresistant promoter [51]. Histology-specific trials are important, helpful, and necessary. Further development of PI3K/AKT/mTOR inhibitors should also consider that single-agent $\mathrm{PI} 3 \mathrm{~K} / \mathrm{AKT} / \mathrm{mTOR}$ pathway inhibition may not always be sufficient to promote response as PIK3CA mutations often coexist with other concurrent molecular alterations. Moreover, predictive biomarkers in order to identify the group of patients with the highest probability to have benefit from this target therapy are still necessary [51].

\section{Conclusion}

The present study reviewed the available evidence regarding the potential impact of some mTOR pathway inhibitors in the treatment of gynecological cancer. Few advances in medical management have occurred in recent years in the treatment of advanced or recurrent gynecological malignancies, and a poor prognosis remains. Rationally designed molecularly targeted therapy is an emerging and important option in this setting; then more investigation in PI3K/AKT/mTOR pathway-targeted therapies is warranted.

\section{Competing Interests}

The authors declare that there is no conflict of interests regarding the publication of this paper.

\section{References}

[1] S. Movva, L. Rodriguez, H. Arias-Pulido, and C. Verschraegen, "Novel chemotherapy approaches for cervical cancer," Cancer, vol. 115, no. 14, pp. 3166-3180, 2009.

[2] N. Husseinzadeh and H. D. Husseinzadeh, "mTOR inhibitors and their clinical application in cervical, endometrial and 
ovarian cancers: A critical review," Gynecologic Oncology, vol. 133, no. 2, pp. 375-381, 2014.

[3] D. Morgensztern and H. L. McLeod, "PI3K/Akt/mTOR pathway as a target for cancer therapy," Anti-Cancer Drugs, vol. 16, no. 8, pp. 797-803, 2005.

[4] S. Chan, "Targeting the mammalian target of rapamycin (mTOR): a new approach to treating cancer," British Journal of Cancer, vol. 91, no. 8, pp. 1420-1424, 2004.

[5] N. Sadeghi and D. E. Gerber, "Targeting the PI3K pathway for cancer therapy," Future Medicinal Chemistry, vol. 4, no. 9, pp. 1153-1169, 2012.

[6] S. H. Advani, "Targeting mTOR pathway: a new concept in cancer therapy," Indian Journal of Medical and Paediatric Oncology, vol. 31, no. 4, pp. 132-136, 2010.

[7] K. D. Courtney, R. B. Corcoran, and J. A. Engelman, “The PI3K pathway as drug target in human cancer," Journal of Clinical Oncology, vol. 28, no. 6, pp. 1075-1083, 2010.

[8] J. LoPiccolo, G. M. Blumenthal, W. B. Bernstein, and P. A. Dennis, "Targeting the PI3K/Akt/mTOR pathway: effective combinations and clinical considerations," Drug Resistance Updates, vol. 11, no. 1-2, pp. 32-50, 2008.

[9] I. Diaz-Padilla, I. Duran, B. A. Clarke, and A. M. Oza, "Biologic rationale and clinical activity of mTOR inhibitors in gynecological cancer," Cancer Treatment Reviews, vol. 38, no. 6, pp. 767$775,2012$.

[10] http://seer.cancer.gov/statfacts/html/corp.html.

[11] http://fundedresearch.cancer.gov/nciportfolio/search/funded? type $=$ site \&fy=PUB2013.

[12] http://www.clinicaltrials.gov.

[13] N. Colombo, C. Creutzberg, F. Amant et al., "ESMO-ESGOESTRO consensus conference on endometrial cancer: diagnosis, treatment and follow-up," Annals of Oncology, vol. 27, no. 1, pp. 16-41, 2016.

[14] NCCN Guidelines v2.2016 for Uterine Cancer, https://www .nccn.org/professionals/physician_gls/pdf/uterine.pdf.

[15] J. V. Bokhman, "Two pathogenetic types of endometrial carcinoma," Gynecologic Oncology, vol. 15, no. 1, pp. 10-17, 1983.

[16] C. Kandoth, N. Schultz, A. D. Cherniack et al., "Integrated genomic characterization of endometrial carcinoma," Nature, vol. 497, pp. 67-73, 2013.

[17] A. M. Oza, L. Elit, M.-S. Tsao et al., "Phase II study of temsirolimus in women with recurrent or metastatic endometrial cancer: a trial of the NCIC Clinical Trials Group," Journal of Clinical Oncology, vol. 29, no. 24, pp. 3278-3285, 2011.

[18] A. M. Oza, S. Pignata, A. Poveda et al., "Randomized phase II trial of ridaforolimus in advanced endometrial carcinoma," Journal of Clinical Oncology, vol. 33, no. 31, pp. 3576-3582, 2015.

[19] S. M. Temkin, S. D. Yamada, and G. F. Fleming, "A phase I study of weekly temsirolimus and topotecan in the treatment of advanced and/or recurrent gynecologic malignancies," Gynecologic Oncology, vol. 117, no. 3, pp. 473-476, 2010.

[20] B. M. Slomovitz, K. H. Lu, T. Johnston et al., "A phase 2 study of the oral mammalian target of rapamycin inhibitor, everolimus, in patients with recurrent endometrial carcinoma," Cancer, vol. 116, no. 23, pp. 5415-5419, 2010.

[21] I. Ray-Coquard, L. Favier, B. Weber et al., "Everolimus as second- or third-line treatment of advanced endometrial cancer: ENDORAD, a phase II trial of GINECO," British Journal of Cancer, vol. 108, no. 9, pp. 1771-1777, 2013.
[22] N. Colombo, D. S. McMeekin, P. E. Schwartz et al., "Ridaforolimus as a single agent in advanced endometrial cancer: results of a single-arm, phase 2 trial," British Journal of Cancer, vol. 108, no. 5, pp. 1021-1026, 2013.

[23] K. Behbakht, M. W. Sill, K. M. Darcy et al., "Phase II trial of the mTOR inhibitor, temsirolimus and evaluation of circulating tumor cells and tumor biomarkers in persistent and recurrent epithelial ovarian and primary peritoneal malignancies: a Gynecologic Oncology Group study," Gynecologic Oncology, vol. 123, no. 1, pp. 19-26, 2011.

[24] U. Matulonis, I. Vergote, F. Backes et al., "Phase II study of the PI3K inhibitor pilaralisib (SAR245408; XL147) in patients with advanced or recurrent endometrial carcinoma," Gynecologic Oncology, vol. 136, no. 2, pp. 246-253, 2015.

[25] A. P. Myers, R. R. Broaddus, V. Makker, P. A. Konstantinopoulos, R. Drapkin, and N. S. Horowitz, "Phase II, two-stage, twoarm, PIK3CA mutation stratified trial of MK-2206 in recurrent endometrial cancer (EC)," Journal of Clinical Oncology, vol. 31, supplement, abstract 5524, 2013, Proceedings of the 2013 ASCO Annual Meeting.

[26] G. F. Fleming, V. L. Filiaci, B. Marzullo et al., "Temsirolimus with or without megestrol acetate and tamoxifen for endometrial cancer: a gynecologic oncology group study," Gynecologic Oncology, vol. 132, no. 3, pp. 585-592, 2014.

[27] B. M. Slomovitz, Y. Jiang, M. S. Yates et al., "Phase II study of everolimus and letrozole in patients with recurrent endometrial carcinoma," Journal of Clinical Oncology, vol. 33, no. 8, pp. 930936, 2015.

[28] P. T. Soliman, S. N. Westin, D. A. Iglesias et al., "Phase II study of everolimus, letrozole, and metformin in women with advanced/recurrent endometrial cancer," Journal of Clinical Oncology, vol. 34, supplement, abstract 5506, 2016, Proceedings of the 2016 ASCO Annual Meeting.

[29] E. A. Alvarez, W. E. Brady, J. L. Walker et al., "Phase II trial of combination bevacizumab and temsirolimus in the treatment of recurrent or persistent endometrial carcinoma: a Gynecologic Oncology Group study," Gynecologic Oncology, vol. 129, no. 1, pp. 22-27, 2013.

[30] M. H. Einstein, R. M. Wenham, and R. Morgan, "Phase II trial of temsirolimus and bevacizumab for initial recurrence of endometrial cancer," Journal of Clinical Oncology, vol. 30, supplement, abstract 5025, 2012, Proceedings of the 2012 ASCO Annual Meeting.

[31] C. Aghajanian, V. L. Filiaci, D. S. Dizon et al., "A randomized phase II study of paclitaxel/carboplatin/bevacizumab, paclitaxel/carboplatin/temsirolimus and ixabepilone/carboplatin/ bevacizumab as initial therapy for measurable stage III or IVA, stage IVB or recurrent endometrial cancer, GOG-86P," Journal of Clinical Oncology, vol. 33, supplement 15, Article ID 5500, 2015.

[32] W. H. Shen, A. S. Balajee, J. Wang et al., "Essential role for nuclear PTEN in maintaining chromosomal integrity," Cell, vol. 128, no. 1, pp. 157-170, 2007.

[33] K. J. Dedes, D. Wetterskog, A. M. Mendes-Pereira et al., "PTEN deficiency in endometrioid endometrial adenocarcinomas predicts sensitivity to PARP inhibitors," Science Translational Medicine, vol. 2, no. 53, p. 53ra75, 2010.

[34] M. D. Forster, K. J. Dedes, S. Sandhu et al., "Treatment with olaparib in a patient with PTEN-deficient endometrioid endometrial cancer," Nature Reviews Clinical Oncology, vol. 8, no. 5, pp. 302-306, 2011. 
[35] R. Siegel, D. Naishadham, and A. Jemal, "Cancer statistics, 2013," CA Cancer Journal for Clinicians, vol. 63, no. 1, pp. 11-30, 2013.

[36] D. B. F. Forman, D. H. Brewster, C. Gombe Mbalawa et al., Eds., GLOBOCAN 2012: Estimated Cancer Incidence, Mortality and Prevalence Worldwide in 2012, 2012, http://www.iarc.fr/.

[37] F. X. Bosch, A. Lorincz, N. Muñoz, C. J. L. M. Meijer, and K. V. Shah, "The causal relation between human papillomavirus and cervical cancer," Journal of Clinical Pathology, vol. 55, no. 4, pp. 244-265, 2002.

[38] B. I. Bertelsen, S. J. Steine, R. Sandvei, A. Molven, and O. D. Laerum, "Molecular analysis of the PI3K-AKT pathway in uterine cervical neoplasia: frequent PIK3CA amplification and AKT phosphorylation," International Journal of Cancer, vol. 118, no. 8, pp. 1877-1883, 2006.

[39] K.-J. Oh, A. Kalinina, N.-H. Park, and S. Bagchi, "Deregulation of eIF4E: 4E-BP1 in differentiated human papillomaviruscontaining cells leads to high levels of expression of the E7 oncoprotein," Journal of Virology, vol. 80, no. 14, pp. 7079-7088, 2006.

[40] I. Beuvink, A. Boulay, S. Fumagalli et al., "The mTOR inhibitor RAD001 sensitizes tumor cells to DNA-damaged induced apoptosis through inhibition of p21 translation," Cell, vol. 120, no. 6, pp. 747-759, 2005.

[41] A. C. de Melo, R. Grazziotin-Reisner, F. Erlich et al., "A phase I study of mTOR inhibitor everolimus in association with cisplatin and radiotherapy for the treatment of locally advanced cervix cancer: PHOENIX I," Cancer Chemotherapy and Pharmacology, vol. 78, no. 1, pp. 101-109, 2016.

[42] J. W. Moroney, M. P. Schlumbrecht, T. Helgason et al., "A phase I trial of liposomal doxorubicin, bevacizumab, and temsirolimus in patients with advanced gynecologic and breast malignancies," Clinical Cancer Research, vol. 17, no. 21, pp. 68406846, 2011.

[43] S. A. Piha-Paul, J. J. Wheler, S. Fu et al., "Advanced gynecologic malignancies treated with a combination of the VEGF inhibitor bevacizumab and the mTOR inhibitor temsirolimus," Oncotarget, vol. 5, no. 7, pp. 1846-1855, 2014.

[44] A. V. Tinker, S. Ellard, S. Welch et al., "Phase II study of temsirolimus (CCI-779) in women with recurrent, unresectable, locally advanced or metastatic carcinoma of the cervix. A trial of the NCIC Clinical Trials Group (NCIC CTG IND 199)," Gynecologic Oncology, vol. 130, no. 2, pp. 269-274, 2013.

[45] Á. H. I. Garces, M. S. F. Dias, E. Paulino, C. G. M. Ferreira, and A. C. De Melo, "Treatment of ovarian cancer beyond chemotherapy: are we hitting the target?" Cancer Chemotherapy and Pharmacology, vol. 75, no. 2, pp. 221-234, 2015.

[46] I. Romero and R. C. Bast Jr., "Minireview: human ovarian cancer: biology, current management, and paths to personalizing therapy," Endocrinology, vol. 153, no. 4, pp. 1593-1602, 2012.

[47] T. Gui and K. Shen, "The epidermal growth factor receptor as a therapeutic target in epithelial ovarian cancer," Cancer Epidemiology, vol. 36, no. 5, pp. 490-496, 2012.

[48] https://www.nccn.org/professionals/physician_gls/pdf/ovarian .pdf.

[49] G. Emons, C. Kurzeder, B. Schmalfeldt et al., "Temsirolimus in women with platinum-refractory/resistant ovarian cancer or advanced/recurrent endometrial carcinoma. A phase II study of the AGO-study group (AGO-GYN8)," Gynecologic Oncology, vol. 140, no. 3, pp. 450-456, 2016.

[50] Available on ECO, European Cancer Observatory. International agency for research on cancer, http://eu-cancer.iarc.fr.
[51] B. Cheaib, A. Auguste, and A. Leary, "The PI3K/Akt/mTOR pathway in ovarian cancer: therapeutic opportunities and challenges," Chinese Journal of Cancer, vol. 34, no. 1, pp. 4-16, 2015.

[52] A. D. Bois, A. Reuss, E. Pujade-Lauraine, P. Harter, I. RayCoquard, and J. Pfisterer, "Role of surgical outcome as prognostic factor in advanced epithelial ovarian cancer: a combined exploratory analysis of 3 prospectively randomized phase 3 multicenter trials: by the arbeitsgemeinschaft gynaekologische onkologie studiengruppe ovarialkarzinom (AGO-OVAR) and the groupe d'Investigateurs nationaux pour les etudes des cancers de l'Ovaire (GINECO)," Cancer, vol. 115, no. 6, pp. 12341244, 2009.

[53] F. Musa, A. Alard, G. David-West, J. P. Curtin, S. V. Blank, and R. J. Schneider, "Dual mTORC1/2 inhibition as a novel strategy for the resensitization and treatment of platinum-resistant ovarian cancer," Molecular Cancer Therapeutics, vol. 15, no. 7, pp. 15571567, 2016.

[54] S. Pignata, L. Cannella, D. Leopardo, C. Pisano, G. S. Bruni, and G. Facchini, "Chemotherapy in epithelial ovarian cancer," Cancer Letters, vol. 303, no. 2, pp. 73-83, 2011.

[55] A. Davis, A. V. Tinker, and M. Friedlander, “"platinum resistant” ovarian cancer: what is it, who to treat and how to measure benefit?" Gynecologic Oncology, vol. 133, no. 3, pp. 624-631, 2014.

[56] T. A. Lawrie, A. Bryant, A. Cameron, E. Gray, and J. Morrison, "Pegylated liposomal doxorubicin for relapsed epithelial ovarian cancer," The Cochrane Database of Systematic Reviews, vol. 7, Article ID CD006910, 2013.

[57] N. Colombo, E. Kutarska, M. Dimopoulos et al., "Randomized, open-label, phase III study comparing patupilone (EPO906) with pegylated liposomal doxorubicin in platinum-refractory or -resistant patients with recurrent epithelial ovarian, primary fallopian tube, or primary peritoneal cancer," Journal of Clinical Oncology, vol. 30, no. 31, pp. 3841-3847, 2012.

[58] J. Sehouli, D. Stengel, P. Harter et al., “Topotecan weekly versus conventional 5-day schedule in patients with platinum-resistant ovarian cancer: a randomized multicenter phase II trial of the North-Eastern German Society of Gynecological Oncology Ovarian Cancer Study Group," Journal of Clinical Oncology, vol. 29, no. 2, pp. 242-248, 2011.

[59] E. Pujade-Lauraine, F. Hilpert, B. Weber et al., "Bevacizumab combined with chemotherapy for platinum-resistant recurrent ovarian cancer: the AURELIA open-label randomized phase III trial," Journal of Clinical Oncology, vol. 32, no. 13, pp. 1302-1308, 2014.

[60] Cancer Genome Atlas Research Network, "Integrated genomic analyses of ovarian carcinoma," Nature, vol. 474, pp. 609-615, 2011.

[61] R. J. Kurman and I.-M. Shih, "Molecular pathogenesis and extraovarian origin of epithelial ovarian cancer-shifting the paradigm," Human Pathology, vol. 42, no. 7, pp. 918-931, 2011.

[62] E. Cerami, J. Gao, U. Dogrusoz et al., "The cBio cancer genomics portal: an open platform for exploring multidimensional cancer genomics data," Cancer Discovery, vol. 2, no. 5, pp. 401-404, 2012.

[63] K. K. Zorn, T. Bonome, L. Gangi et al., “Gene expression profiles of serous, endometrioid, and clear cell subtypes of ovarian and endometrial cancer," Clinical Cancer Research, vol. 11, no. 18, pp. 6422-6430, 2005.

[64] D. A. Levine, F. Bogomolniy, C. J. Yee et al., "Frequent mutation of the PIK3CA gene in ovarian and breast cancers," Clinical Cancer Research, vol. 11, no. 8, pp. 2875-2878, 2005. 
[65] F. Janku, J. J. Wheler, S. N. Westin et al., "PI3K/AKT/mTOR inhibitors in patients with breast and gynecologic malignancies harboring PIK3CA mutations," Journal of Clinical Oncology, vol. 30, no. 8, pp. 777-782, 2012.

[66] S. Mabuchi, H. Kuroda, R. Takahashi, and T. Sasano, "The $\mathrm{PI} 3 \mathrm{~K} / \mathrm{AKT} / \mathrm{mTOR}$ pathway as a therapeutic target in ovarian cancer," Gynecologic Oncology, vol. 137, no. 1, pp. 173-179, 2015.

[67] P. Andorfer, A. Heuwieser, A. Heinzel, A. Lukas, B. Mayer, and P. Perco, "Vascular endothelial growth factor A as predictive marker for mTOR inhibition in relapsing high-grade serous ovarian cancer," BMC Systems Biology, vol. 10, article no. 33, 2016.

[68] S. Mabuchi, C. Kawase, D. A. Altomare et al., "mTOR is a promising therapeutic target both in cisplatin-sensitive and cisplatin-resistant clear cell carcinoma of the ovary," Clinical Cancer Research, vol. 15, no. 17, pp. 5404-5413, 2009.

[69] F. Di Nicolantonio, S. Arena, J. Tabernero et al., "Deregulation of the PI3K and KRAS signaling pathways in human cancer cells determines their response to everolimus," The Journal of Clinical Investigation, vol. 120, no. 8, pp. 2858-2866, 2010.

[70] E. Stover, L. A. Garraway, and U. A. Matulonis, "Somatic alterations in the PTEN-PI3K-AKT-mTOR pathway in a cohort of 379 ovarian cancer patients," Journal of Clinical Oncology, vol. 34, supplement, abstract 5572, 2016.

[71] H. Itamochi and J. Kigawa, "Clinical trials and future potential of targeted therapy for ovarian cancer," International Journal of Clinical Oncology, vol. 17, no. 5, pp. 430-440, 2012.

[72] M. Takano, Y. Kikuchi, K. Kudoh et al., "Weekly administration of temsirolimus for heavily pretreated patients with clear cell carcinoma of the ovary: a report of six cases," International Journal of Clinical Oncology, vol. 16, no. 5, pp. 605-609, 2011.

[73] M. D. I. Boers-Sonderen, I. Desar, W. T. A. Van Der Graaf, P. B. Ottevanger, and C. Van Herpen, "A phase Ib study of the combination of temsirolimus and pegylated liposomal doxorubcin in advanced or recurrent breast, endometrial and ovarian cancer," Journal of Clinical Oncology, vol. 30, supplement, abstract 5061, 2012, Proceedings of the 2012 ASCO Annual Meeting.

[74] C. Kollmannsberger, H. Hirte, L. L. Siu et al., "Temsirolimus in combination with carboplatin and paclitaxel in patients with advanced solid tumors: a NCIC-CTG, phase I, open-label doseescalation study (IND 179)," Annals of Oncology, vol. 23, no. 1, Article ID mdr063, pp. 238-244, 2012.

[75] H. J. P. Chon, P. L. Judson, S. Apte, J. H. Lee, I. Williams-Elson, and R. M. Wenham, "Phase I study of combination carboplatin, paclitaxel and ridaforolimus in patients with solid, endometrial and ovarian cancers," Journal of Clinical Oncology, vol. 32, abstr 2614, 2014.

[76] M. Campone, V. Levy, E. Bourbouloux et al., "Safety and pharmacokinetics of paclitaxel and the oral mTOR inhibitor everolimus in advanced solid tumours," British Journal of Cancer, vol. 100, no. 2, pp. 315-321, 2009.

[77] M. K. H. Takano, H. Kouta, Y. Ikeda et al., "Combination chemotherapy with temsirolimus and trabectedin for recurrent clear cell carcinoma of the ovary: a phase II single arm clinical trial," Journal of Clinical Oncology, vol. 32, abstract 5517, 2014.

[78] A. P. Myers, "New strategies in endometrial cancer: targeting the PI3K/mTOR pathway-the devil is in the details," Clinical Cancer Research, vol. 19, no. 19, pp. 5264-5274, 2013. 


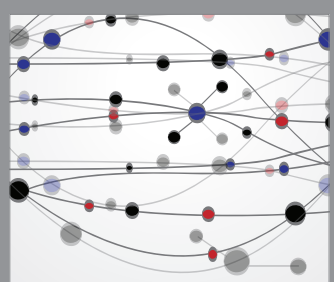

The Scientific World Journal
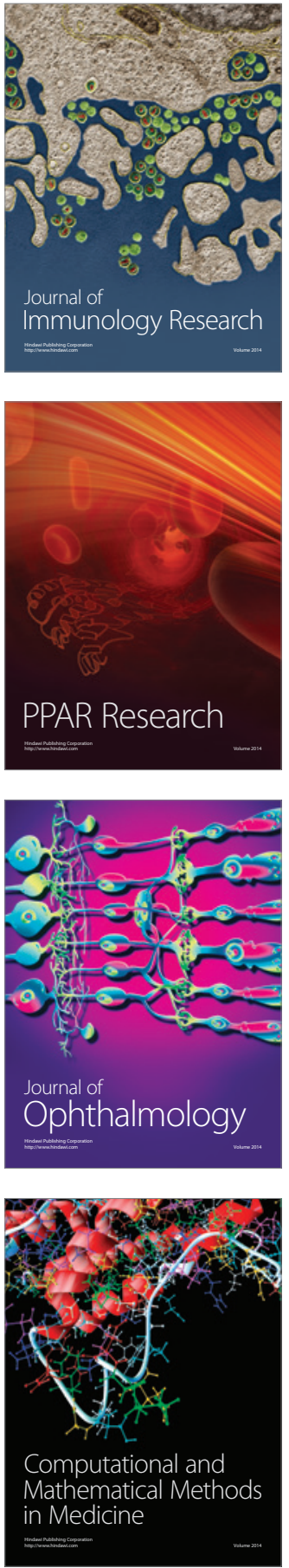

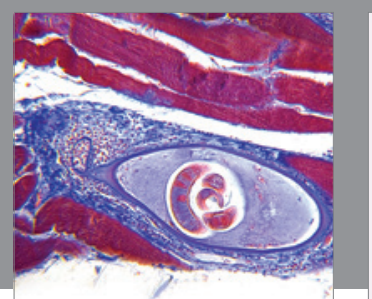

Gastroenterology Research and Practice
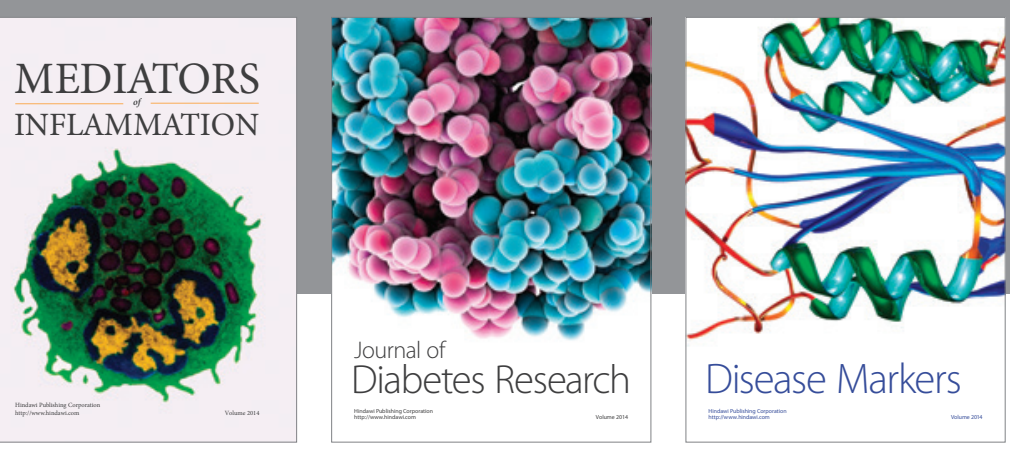

Disease Markers

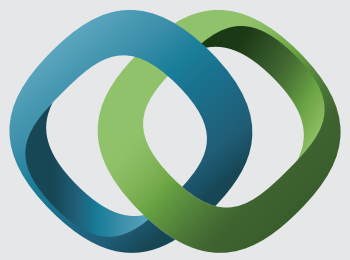

\section{Hindawi}

Submit your manuscripts at

https://www.hindawi.com
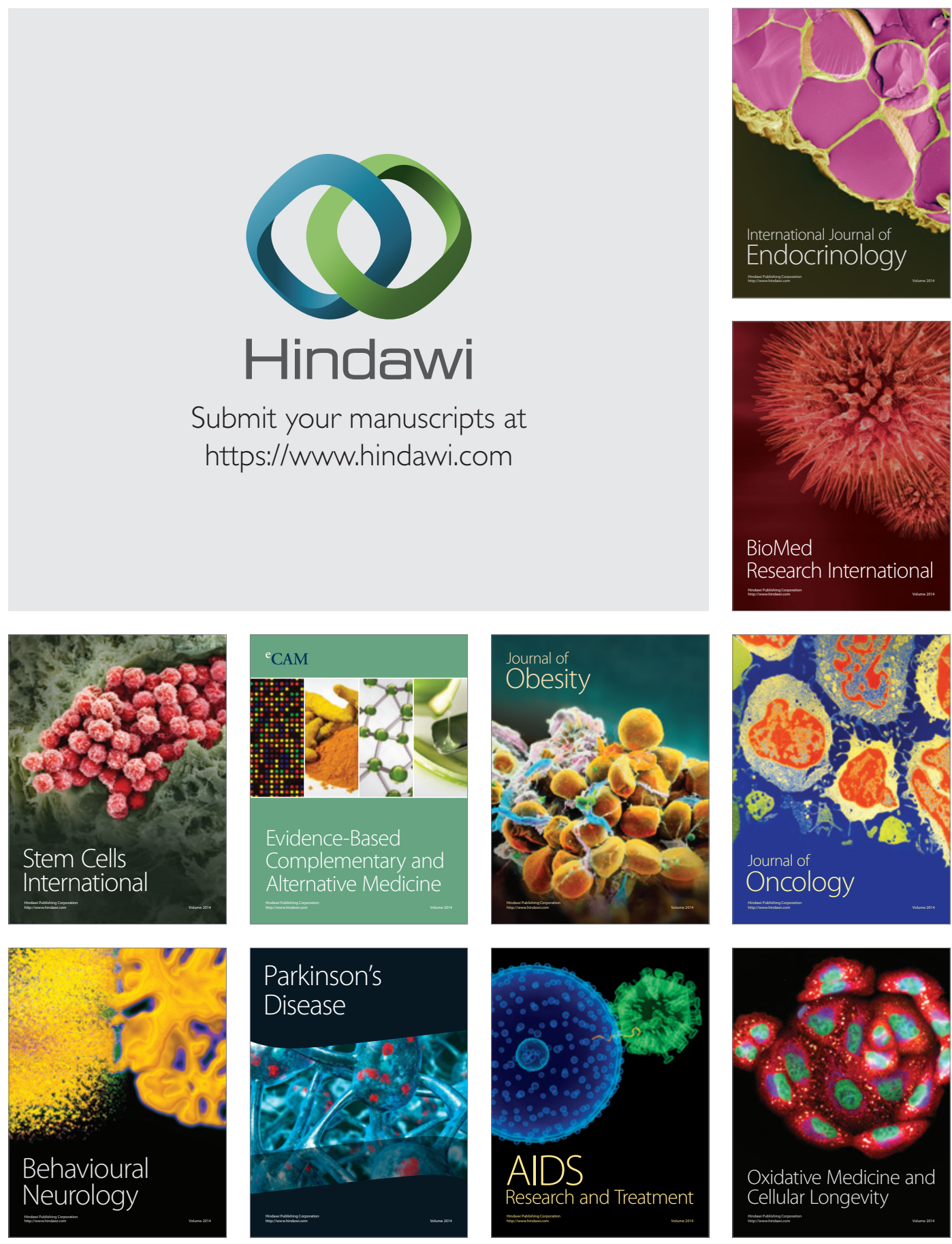